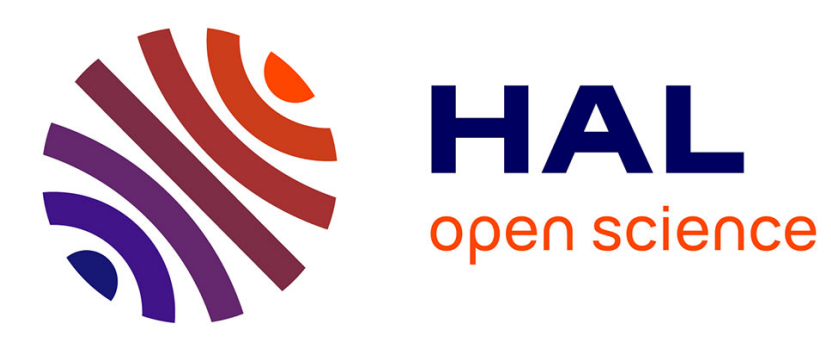

\title{
A Numerical Model for Miscible Displacement of Multi-Component Reactive Species
}

\author{
Mohammed Adil Sbai, Mohamed Azaroual
}

\section{To cite this version:}

Mohammed Adil Sbai, Mohamed Azaroual. A Numerical Model for Miscible Displacement of MultiComponent Reactive Species. 15th International Conference on Computational Methods in Water Resources (CMWR XV), Jun 2004, Chapel Hill, NC, United States. pp.510-520. hal-00589804

\section{HAL Id: hal-00589804 https: / hal-brgm.archives-ouvertes.fr/hal-00589804}

Submitted on 2 May 2011

HAL is a multi-disciplinary open access archive for the deposit and dissemination of scientific research documents, whether they are published or not. The documents may come from teaching and research institutions in France or abroad, or from public or private research centers.
L'archive ouverte pluridisciplinaire HAL, est destinée au dépôt et à la diffusion de documents scientifiques de niveau recherche, publiés ou non, émanant des établissements d'enseignement et de recherche français ou étrangers, des laboratoires publics ou privés. 


\title{
A Numerical Model for Miscible Displacement of Multi-Component Reactive Species
}

\author{
M. A. Sbai ${ }^{a}$ and M. Azaroual ${ }^{a}$ \\ ${ }^{a}$ Water Department/Groundwater and Geochemistry Modeling, BRGM (French \\ Geological Survey) \\ 3, Avenue C. Guillemin, BP 6009, 45060 Orléans Cedex 2, France \\ e-mail(s): a.sbai@brgm.fr, a.azaroual@brgm.fr
}

The study of instable miscible displacement of chemical species is an important process for many environmental and reservoir engineering applications. A new coupled saturated porous media flow and reactive transport numerical model: RTAFF, has been developed for the assessment and analysis of complex feedback loops between physical transport, geochemistry, and fluid flows in such environments.

The model accounts for changes of flowing phase mobility upon viscosity of individual species in the aqueous solution, and field permeability attributed mainly to mineral precipitation-dissolution reactions. An operator splitting framework sequentially couples a mixed-hybrid finite element flow model on quadrilateral/hexahedral grids, with a mass conservative advective-dispersive-reactive (ADR) model able to handle a full range of homogeneous and heterogeneous reactions, using a mixed kinetic equilibrium approach. The multi-component species ADR simulator workflow involves a time-split procedure adapted for each single process solver, thus advection and dispersion operators are also solved independently using other specialized computer models. Example applications are presented in one, and two space dimensions, showing the effectiveness of the numerical model for practical environmental applications. First results demonstrate the accuracy, efficiency and robustness of our approach. Therefore, the model may prove useful for many practical applications.

\section{INTRODUCTION}

Demands to undertake modeling analysis of coupled groundwater flow, solute transport, and reactive water-rock interactions, is increasing among scientists and engineers in different disciplines. The development of numerical models for coupled subsurface phenomena is growing considerably, as current literature attests $[6,10,11,15,14,17]$. Several numerical difficulties may arise when many interacting phases, like dissolved aqueous species, immobile adsorbed species, and precipitation / dissolution of minerals and gases, are taken simultaneously, and are affecting each other. Concentrations of chemical species, even for those present in small amounts, must be kept positive. Mass conservation has to be verified locally for each grid cell. Non-oscillatory behavior for highly advective flows should be suppressed, to accurately capture physical sharp moving fronts of, aqueous components, 
and mineral phases. Therefore, stringent requirements are needed on the performances of numerical techniques.

In this paper we present RTAFF, which is a new reactive transport model developed recently. In the process of designing the simulator, several issues related to (i) robustness, (ii) accuracy, and (iii) efficiency are considered. Another focus is to illustrate the challenges and difficulties in applying the presented numerical algorithms to practical problems. Realistic applications involve quite often multi-dimensional domains, highly heterogeneous permeability fields, and reactions spanning different time scales.

\section{MATHEMATICAL MODEL}

The mathematical model is a combination of three modules for, groundwater flow, solute transport, and batch geochemistry. An overview of the governing equations, with the underlying approximations taken are briefly presented in the subsections below. A complete description of the model should be found elsewhere [11].

\subsection{Groundwater flow}

The governing equations for three-dimensional, saturated, incompressible, groundwater flow are derived from the (i) mass conservation, and (ii) generalized Darcy's laws, given as (see $[2,13]$ for more details)

$$
\begin{aligned}
& S_{0} \frac{\partial \psi}{\partial t}+\nabla \cdot \mathbf{u}=q \\
& \mathbf{u}=-\frac{\mathbf{k}}{\mu}[\nabla \psi-\rho g \nabla z]
\end{aligned}
$$

where $S_{0}$ is the specific storage coefficient $\left[\mathrm{L}^{-1}\right], \psi$ is the pressure potential $[\mathrm{L}], \mathbf{u}$ is the Darcy velocity vector $[\mathrm{L} / \mathrm{T}], q$ is the external source and/or sink term $\left[\mathrm{T}^{-1}\right], \mathbf{k}$ is the permeability tensor $\left[\mathrm{L}^{2}\right], \mu$ is the dynamic fluid viscosity $[\mathrm{M} / \mathrm{LT}], g$ is the acceleration due to gravity $\left[\mathrm{L}^{2} / \mathrm{T}\right], \rho$ is the fluid density $\left[\mathrm{M} / \mathrm{L}^{3}\right], \nabla=\left(\frac{\partial}{\partial x}, \frac{\partial}{\partial y}, \frac{\partial}{\partial z}\right)^{T}$ is the del operator $\left[\mathrm{L}^{-1}\right]$, where $\mathbf{x}=(x, y, z)^{T}$ is the location vector $[\mathrm{L}]$, the $z$-axis is taken vertical and positive downwards, and $t$ is time $[\mathrm{T}]$.

\subsection{Reactive geochemical transport}

Mass conservation laws in saturated porous media, for a multi-component reactive system, yield the following nonlinear partial differential equations (see $[6,15,17]$ for more details)

$$
\frac{\partial\left(\phi U_{j}\right)}{\partial t}+\nabla \cdot\left[\mathbf{J}_{A d}+\mathbf{J}_{D i s p}\right]=\mathcal{R}_{j}^{K}\left(C_{1}, \ldots, C_{N_{S}}\right)+q_{j} \quad j=1, \ldots, N_{C}
$$

where, $N_{S}$ is the total number of species, divided into $N_{C}$ primary basis species and $N_{R}$ secondary species set, e.g. the number of independent chemical reactions [6], $\phi$ is the porosity, $C_{i}$ is the concentration of species $i\left[\right.$ moles $\left./ \mathrm{L}^{3}\right], U_{j}$ is the total aqueous concentration of primary species $j$ in solution $\left[\right.$ moles $\left./ \mathrm{L}^{3}\right], \mathbf{J}_{A d}=\mathbf{u} U_{j}$ and $\mathbf{J}_{D i s p}=-\mathbf{D} \nabla U_{j}$, are the 
advective, and dispersive fluxes respectively [moles $\left./ \mathrm{L}^{2} \mathrm{~T}\right], \mathcal{R}_{j}^{K}=\frac{d C_{j}}{d t}$ is the total reaction rate of all kinetic reactions involving species $j\left[\right.$ moles $\left./ \mathrm{L}^{3} \mathrm{~T}\right], \mathbf{D}$ is the hydrodynamic dispersion tensor [2], including the effects of molecular diffusion coefficient, longitudinal, and transverse dispersions $\left[\mathrm{L}^{2} / \mathrm{T}\right] . q_{j}$ is the source and/or sink term $\left[\right.$ moles $\left./ \mathrm{L}^{3} \mathrm{~T}\right]$ representing injection or production wells, and expressed as

$$
q_{j}=|q| U_{j}^{e x t}-|q| U_{j}
$$

where, $|q|$ is the absolute value of the positive/negative injection/extraction flow rate, and $U_{j}^{e x t}$ is an external total concentration.

Chemical reactions are either sufficiently fast, allowing for a thermodynamic equilibrium description, or rate-limited. Examples of the $N_{R}^{Q}$ equilibrium reactions, are the homogeneous aqueous phase, complexation reactions, or surface complexation reactions. Heterogeneous aqueous-mineral reactions, are part of the $N_{R}^{K}$ kinetic, or rate-limited reactions. Notice that, $N_{R}=N_{R}^{Q}+N_{R}^{K}$. Total concentrations are defined as:

$$
U_{j}=C_{j}+\sum_{i=1}^{N_{R}^{Q}} \nu_{j, i+N_{C}} C_{i+N_{C}} \quad j=1, \ldots, N_{C}
$$

where, $\nu_{j, i}$ is the stoichiometric coefficient for species $i$ in component $j$. Concentrations of secondary aqueous species are determined from mass action laws:

$$
\left\{\begin{array}{l}
\log a_{i+N_{C}}=\sum_{j=1}^{N_{C}} \nu_{j, i} \log a_{j}+\log K_{i} \quad i=1, \ldots, N_{R}^{Q} \\
\log a_{i}=\log \gamma_{i}+\log C_{i} \quad i=1, \ldots, N_{C}+N_{R}^{Q}
\end{array}\right.
$$

where, $a_{i}$ and $\gamma_{i}$ are the molal activity and activity coefficient of species $i$ respectively. We use Davies equation [9] for low ionic strength solutions, $I$, and given by

$$
\log \gamma_{i}=-A z_{i}^{2}\left(\frac{\sqrt{I}}{1+\sqrt{I}}-0.3 I\right)
$$

where, $z_{i}$ is the ionic charge of aqueous species $i, A$ is a temperature dependent coefficient, and the ionic strength is $I=\frac{1}{2} \sum_{i=1}^{N_{a q}} z_{i}^{2} C_{i}$. Davies equation links the activity coefficients with the concentrations of aqueous species. Concentrations of equilibrium mineral phases are given by:

$$
\begin{cases}\sum_{j=1}^{N_{C}} \nu_{j, i} \log a_{j}+\log K_{i}=0 & \text { if } a_{i+N_{C}}>0 \\ \sum_{j=1}^{N_{C}} \nu_{j, i} \log a_{j}+\log K_{i} \leqslant 0 & \text { if } a_{i+N_{C}}=0\end{cases}
$$

It is interesting to highlight the non differentiable character of the nonlinear algebraic equilibrium equations 6 and 8. When kinetics are involved, the problem becomes of coupled differential algebraic nature $[15,14,17]$. 


\subsection{Coupled flow and reactive transport}

Groundwater flow is coupled with reactive transport to allow for simulation of miscible displacement processes in contaminant transport, and reservoir engineering applications. In this case, we allow a variable mixture viscosity in the form:

$$
\mu=F\left[\mu_{0},\left.\chi_{i}\right|_{i=1, \ldots, N_{S}},\left(\frac{\mu_{i}}{\mu_{0}}\right)_{i=1, \ldots, N_{S}}\right]
$$

In general the function $F$ depends on the resident fluid viscosity $\mu_{0}$, the mass fraction of individual species in solution, $\chi_{i}=C_{i} M_{i} / \rho$, where $M_{i}$ is the molecular weight of species $i$, and $\mu_{i} / \mu_{0}$ is the viscosity ratio or the inverse of the mobility ratio.

Such expressions are given as a fifth order polynomial at given temperature and pressure values, or as a power quarter mixing rule [1]. The later is traditionally used in petroleum reservoir engineering, and expressed as

$$
\frac{\mu}{\mu_{0}}=\left[\sum_{i=0}^{N_{S}}\left(\frac{\mu_{i}}{\mu_{0}}\right)^{-1 / 4} \chi_{i}\right]^{-4}
$$

\section{THE NUMERICAL MODEL}

Solving the coupled set of equations 1 and 3, subject to equations 2, 5, 6 and 8 in one step is notoriously a complex task for multi-dimensional problems. Although its feasibility has been demonstrated by several researchers (see [15] and the references therein) it remains of very high CPU cost and not suitable for integration of independent evolving computer codes. Therefore, in this study operator-splitting methods are preferred, because they enable (a) tight integration of different specialized computer models in the same framework, (b) breaking down the problem nonlinearity by isolating the geochemical batch computations at each time step, (c) and making it possible to use high Péclet number algorithms, like high resolution finite volume methods [16] (HRFV). As already stated by [14], the use of shock-capturing HRFV methods may be considered as the chief advantage of operator-splitting techniques.

\subsection{Discretization techniques and numerical methods}

The numerical model hierarchy includes four integrated modules:

\subsubsection{Mixed Hybrid Finite Element (MHFE) Flow Module}

The flow model is approximated by either quadrilateral or hexahedral elements of general shape. A mixed hybrid finite element approximation solves for pressure heads simultaneously at cells centers and edges of the mesh. In a post-processing step the numerical fluxes at the mesh faces are recovered. Constant RT0 (Raviart-Thomas) scalar basis functions are used for approximating the pressure head at the cell center, while vector basis functions are associated with the edge fluxes. The time discretization scheme is fully implicit, and enhanced by the use of an efficient sparse incomplete factorization preconditioned conjugate gradient solver [13]. The model supports distributed cell-centered parameters for all material properties, full tensor quantities, and common boundary conditions for hydrogeological applications. Recently, it has been integrated in a modern pre- 
and post- processing environment, where several automatic mesh generator packages with mesh refinement and general geometry fitted possibilities are also available.

\subsubsection{High Resolution Finite Volume (HRFV) Advection Module}

Adective transport is approximated by an explicit cell-centered higher order Godunov method. This is basically a two-step method. First, an approximate Riemann solver [16] computes new conservative fluxes at cell-interfaces, from cell-centered concentrations at the previous time step. Next, a nonlinear reconstruction method with appropriate slope limiters is used to prevent oscillatory behavior near the sharp fronts. The threedimensional slope limiting function prevents local minima and maxima to rise below and above their respective values at the previous time level. Therefore, undershoots or/and overshoots do not arise during the solution procedure. This is of great importance for the next succeeding reaction step in the time splitting algorithm.

The numerical scheme allows for high Péclet numbers to be taken, and thus even coarse meshes exhibit only a small amount of numerical diffusion. This however, comes at price of a Courant-Friedrich-Levy (CFL) constraint limiting time step size, which is enforced directly in the computer model.

\subsubsection{Batch geochemical reactor Module}

A new geochemistry module, developed from scratch [12] and based on mixed equilibriumkinetic formulation, is beginning to prove efficient and robust in coupled mode. The thermodynamic equilibrium batch engine uses robust constrained optimization techniques [8], such as the interior points method [5] to minimize the Gibbs free energy function, subject to mass-balance and positive activities constraints. The model also enables simulation of biodegradation kinetics using various Monod rate law forms.

\subsubsection{Dispersion-diffusion Module}

Dispersion is approximated again by the MHFE module. Here we just reuse the flow solver, with modified tensor and scalar variables. All transport parameters, such as dispersivities, and molecular diffusion may be given as cell-centered distributed data sets.

\subsection{Time splitting algorithm}

The ADR equations are solved using an adaptive time splitting algorithm, which occupies a central place in the computer code framework. This algorithm accounts for the possible failure of any module. When this happens we do back stepping with a smaller time step size. Let $\tau_{A d v}$ and $\tau_{R}$ be the number of sub-steps for solving the advection and reaction terms for a given reactive time step $\Delta t$ respectively, and $k$ the current time step level. The algorithm proceeds as follows:

1. Advection operator:

- Determine the optimum time step $\Delta t_{C F L}$ for the mesh, guarantying the CFL time step constraint, and hence $\tau_{a d v}$.

For $1, \ldots, \tau_{a d v}$ solve explicitly the system:

$$
\phi \frac{U_{j}^{k, a d v}-U_{j}^{k}}{\Delta t / \tau_{a d v}}=\nabla \cdot U_{j}^{k} \mathbf{u}^{k}+q_{j} \quad \text { for } j=1, \ldots, N_{C}
$$


2. Reaction operator:

- For $1, \ldots, \tau_{R}$

solve the algebraic-differential system:

$$
\frac{U_{j}^{k, R}-U_{j}^{k, a d v}}{\Delta t / \tau_{R}}=\mathcal{R}_{j}^{K}\left(C_{1}^{k, a d v}, \ldots C_{N_{C}}^{k, a d v}, C_{N_{C}+1}^{k} \ldots, C_{N_{S}}^{k}\right) \text { for } j=1, \ldots, N_{C}
$$

if convergence is not reached for a given reactor, then

$$
\Delta t=\Delta t / 2, \text { goto } 1
$$

3. Dispersion-diffusion operator:

- solve implicitly the system:

$$
\phi \frac{U_{j}^{k, d i s p}-U_{j}^{k, R}}{\Delta t}=\nabla \cdot \mathbf{D}^{k} \nabla U_{j}^{k, d i s p} \text { for } j=1, \ldots, N_{C}
$$

4. Speciation and Equilibrium step:

- The aqueous composition is equilibrated using the chemical equilibrium operator $\mathcal{E} \mathcal{Q}$ exclusively:

$$
U_{j}^{k+1}=\mathcal{E} \mathcal{Q}\left(C_{1}^{k, d i s p}, \ldots C_{N_{C}}^{k, d i s p}, C_{N_{C}+1}^{k+1} \ldots, C_{N_{S}}^{k+1}\right) \text { for } j=1, \ldots, N_{C}
$$

We point out that looping over the grid cells is internal to the advection routine, and external to the reaction step. This is enforced to enhance data locality, such that little code restructuring will be needed in distributed computing tasks. Porting the presented algorithms to parallel computer platforms is a future extension.

\section{DEMONSTRATION EXAMPLES}

Since model development begun, in late 2002, several test problems were designed for verifications purposes. Some simple one-dimensional models, as the first example shown below, were compared with the results of other available models, to establish the accuracy, and eventually the efficiency of the implemented algorithms [12]. Based on this first knowledge, it is trusted that the computer model, in its present state, is able to handle complex coupled mechanistic processes, of fluid flow, and reactive geochemistry, in multi-dimensional heterogeneous domains. Many ongoing research efforts, under several integrated projects, will be using the developed model as a tool for investigating environmental, and groundwater pollution problems. 


\subsection{One-dimensional verification example}

This benchmark problem was designed to assess the model capabilities to account simultaneously for combined aqueous complexation, and dissolution / precipitation reactions, including oxidation / reduction reactions. First, a comparative study with PHREEQC computer model [10] is performed. A numerical grid convergence study follows.

A rich sulfuric acid solution corresponding to an initial $\mathrm{pH}$ of 4 is continuously injected at the upstream boundary of a soil column. The problem domain is a $5 \mathrm{dm}$ length, homogeneous, and composed mainly from gypsum, and calcite minerals. Initial and boundary conditions, as well as other simulation parameters are given in table 1 .

\begin{tabular}{lll}
\hline Component & Initial conditions (mol/l) & Boundary conditions (mol/l) \\
\hline $\mathrm{S}(-\mathrm{II})$ & 0 & $1 \mathrm{E}-3$ \\
$\mathrm{~S}(+\mathrm{VI})$ & 0 & 0 \\
$\mathrm{C}(+\mathrm{IV})$ & $1 \mathrm{E}-4$ & $5 \mathrm{E}-6$ \\
$\mathrm{Ca}$ & $1 \mathrm{E}-4$ & $5 \mathrm{E}-6$ \\
$\mathrm{pH}$ & 13 & 4 \\
$\mathrm{pE}$ & 4 & 4 \\
Calcite & 0 & - \\
Anydrite & 0 & - \\
Gypse & 0.1 & - \\
\hline
\end{tabular}

Table 1

Initial and boundary conditions for example 1.

A pore water velocity of $1.6 \mathrm{E}-6 \mathrm{~m} / \mathrm{s}$ is maintained constant along the simulation run, such that the flow field is not computed. This problem involves $N_{C}=6$ number of chemical components, and $N_{R}=N_{R}^{Q}=13$ independent chemical reactions. In both models, thermodynamic data is taken unmodified from the LLNL database file distributed with PHREEQC [10].

Numerical simulations were run on uniform grids of 500 and 250 cells, for the two models PHREEQC and RTAFF respectively. Other simulation parameters of interest are: $\alpha_{L}=0.0067 m, \phi=0.32, t_{\text {final }}=84000$ seconds, and CFL $=0.5$.

Simulations showed a sequence of precipitation and dissolution of calcite. As shown in Figure 1B this zone is very narrow, and is represented by the pic concentration. This is a classical result of the strong $\mathrm{pH}$ increase downward the flow gradient. The spatial $\mathrm{pH}$ zone favorable to a wide precipitation area of calcite is restricted. There is a very good agreement between the results obtained with the two models. An interesting fact to mention however, is that the 250 cells simulation was intractable with PHREEQC model.

Within RTAFF code, this simulation was run in 421 reactive time steps (the first corresponding to aqueous speciation), since the operator-splitting algorithm has computed a $\Delta t_{C F L}=200$ seconds for all time steps. A total CPU time of 14 min20s was needed on a mobile AMD Athlon XP computer, of $1.4 \mathrm{GHz}$, and with 512MB RAM. 

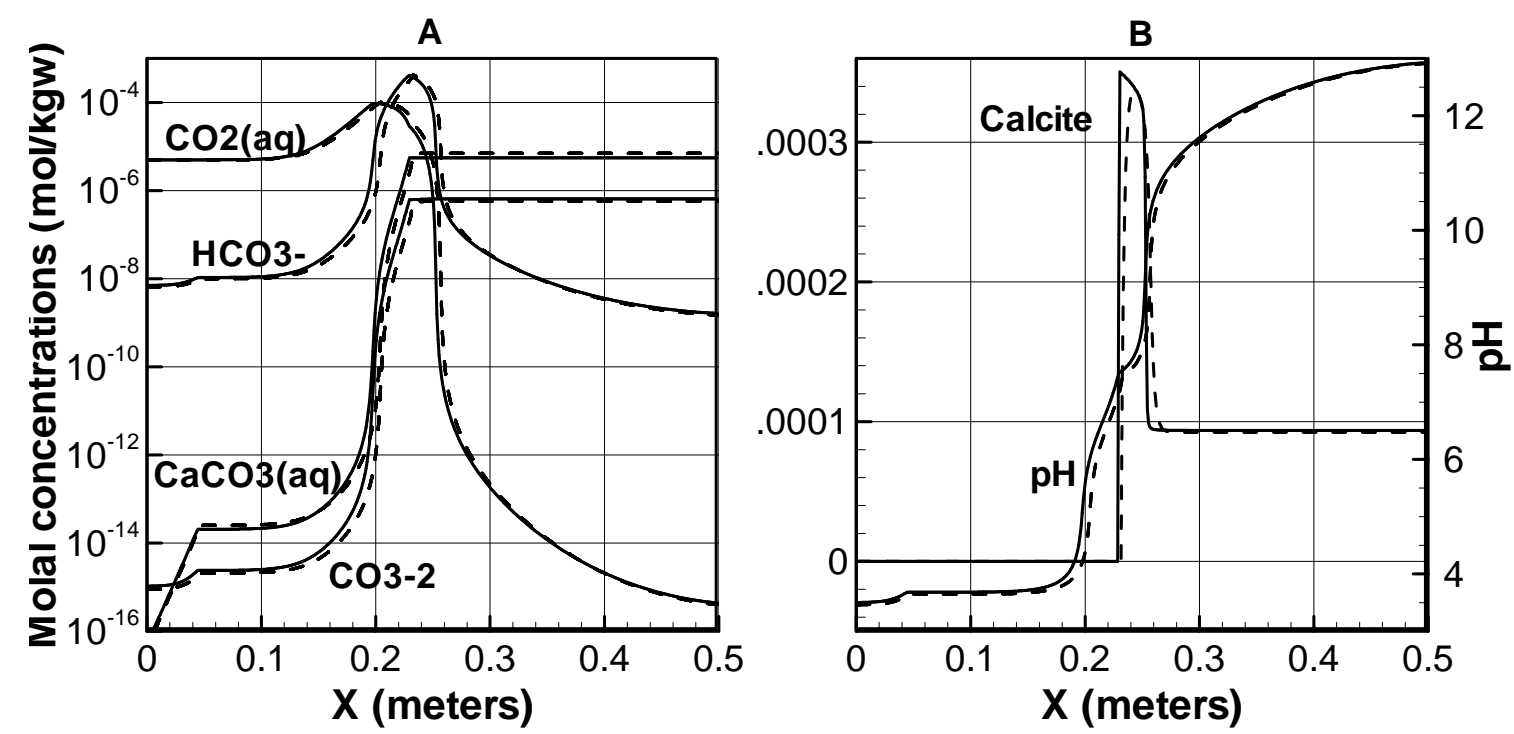

Figure 1. Comparison between PHREEQC results (solid lines) and RTAFF results (dashed lines), for (A) some carbonate aqueous species, (B) calcite amount (in moles) and $\mathrm{pH}$, at 84000 seconds.

\subsection{Acid gas migration}

Acid gas injection in deep saline aquifers, and hydrocarbon reservoirs is presented as a promising way to reduce greenhouse gas emissions into the atmosphere. Geological sequestration options of carbon dioxide, and other greenhouse gases, are therefore studied by numerical models. This demonstration examples aims to show that the developed model, could be useful in understanding the involved fluid-gas-rock interactions.

A two-dimensional $[0,100] \times[0,100]$ meters domain is divided into a $100 \times 100$ uniform grid. A stochastic permeability distribution is obtained with the HYDRO_GEN [3] field generator, with an average value of $2 \mathrm{~m}$ /day, and a correlation length of $5 \mathrm{~m}$, that is the size of 5 cells. The initial geochemical composition, as well as for the recharging groundwater from the left boundary, are identical to the ones described in table 1. Other relevant parameters for this problem are: $D_{0}=10^{-9} \mathrm{~m}^{2} / \mathrm{s}, \phi=0.4, t_{\text {final }}=50$ days, and $\mathrm{CFL}=0.5$. The effects of macrodispersion are deliberately eliminated, creating highly advective flow in permeable zones, and diffusion controlled transport in clogged pores.

Figures 2 and 3 illustrate the role of field heterogeneities in triggering displacement instabilities. Fingered displacement of reactive species occurs with different and contrasted mobilities. Even when field dispersion is neglected, the concentrations of some species are not anymore front controlled, as the intuition would suggest.

By contract to the one dimensional example, small gray spots in figure $2 \mathrm{~B}$ indicate disparate zones of spatial calcite distribution. These zones are non-uniformly distributed. Other simulations scenarios conducted by including dispersion, indicate that they grow up with increasing dispersivities and mixing. This first result would suggest that sequestration in mineral form predicted by lower dimensional models would be not sufficient enough for other spatial scales. 
(A)

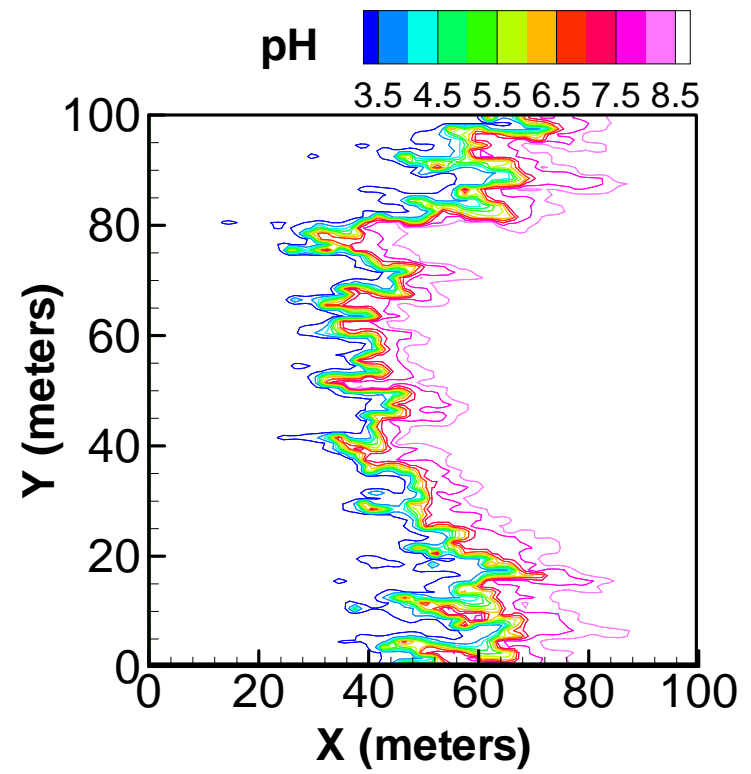

(B)

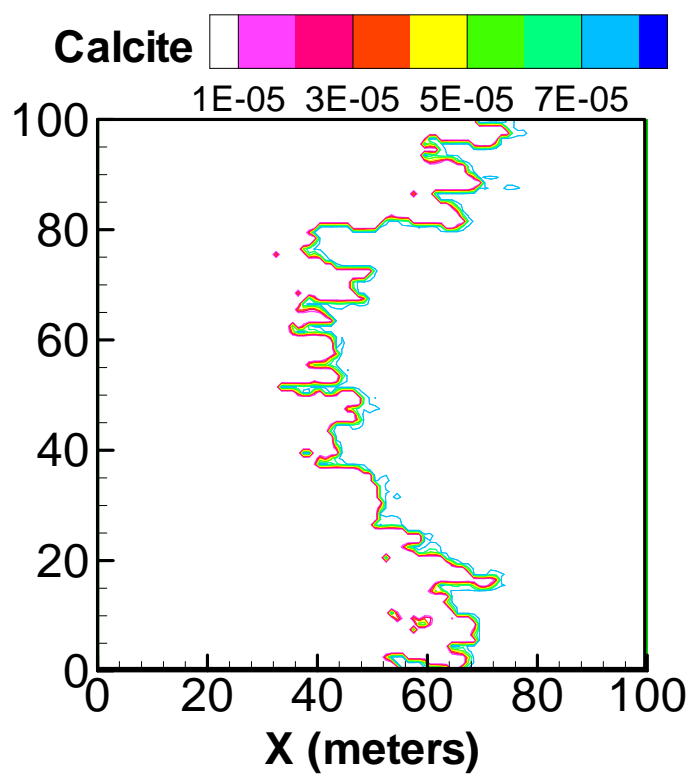

Figure 2. $\mathrm{pH}$ and calcite mineral fronts after 20 days.

(A)

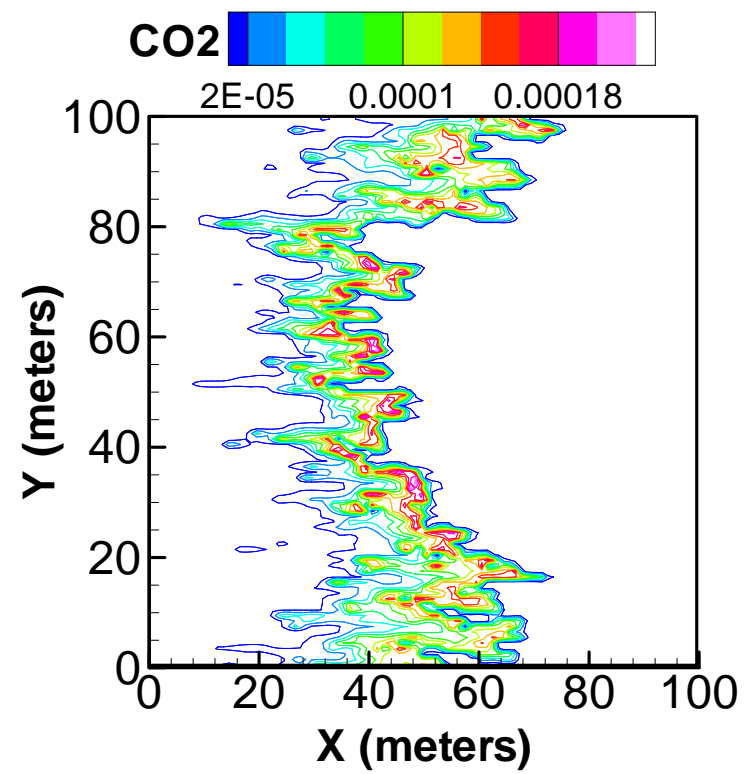

(B)

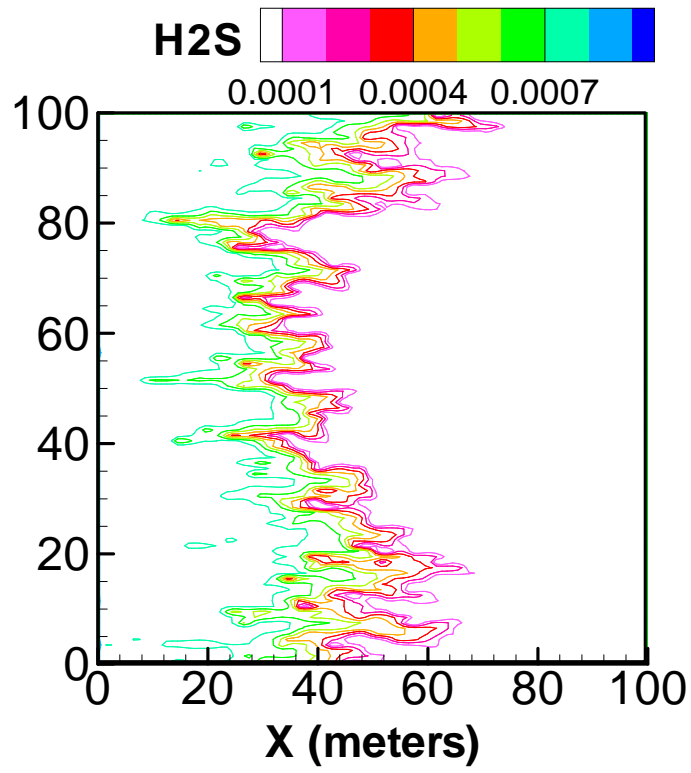

Figure 3. Distribution of aqueous $\mathrm{CO}_{2}$ and $\mathrm{H}_{2} \mathrm{~S}$ molal after 20 days.

Another remarkable result is that, another series of simulations have showed the dominant role of field permeability in controlling the dissolved species displacement. By increasing $\mathrm{CO}_{2}$ viscosity ratio to a factor of 40 , which is representative for $\mathrm{CO}_{2}$ disposal conditions at depths below 800 meters (see [4] and references therein), we notice negligible changes in the distribution of all species.

A typical simulation was run in 1417 reactive time steps, 217 time step cuts were necessary to capture the fastest fronts. A minimum time step of $9.6 \mathrm{E}-4$ days was reached, 
and a total CPU time of $1 \mathrm{~h} 35 \mathrm{~min} 17 \mathrm{~s}$ was needed, among which $1 \mathrm{~h} 0 \mathrm{~min} 35 \mathrm{~s}$ CPU was devoted to the reactive time steps (on the same platform as in the first example).

The results showed here are so far preliminary. Indeed, the possible improvements may include, a kinetic parameterization of the slow mineral reactions, taking into account a more detailed speciation, and perform sensitivity analysis with respect to other key transport parameters.

\section{CONCLUSIONS}

In this study, a new numerical model has been developed for an accurate description of mechanistic coupled processes of, fluid flows, solute transport, and chemical reactions. The computer code is made extensible through efficient and flexible operator time splitting techniques, integrating several specialized modules, and adapted internally for each single operator. These models use different approximation techniques including, the HRFV based advection, and the MHFE dispersion-diffusion. Numerical experiments, and other code comparison studies, have shown the efficiency of the proposed approaches. Sharp aqueous and mineral fronts are accurately captured, and species concentrations are stabilized to remain positive during the solution procedure. Theses results suggests that the numerical model, may be applied to several groundwater pollution, and environmental assessment problems.

\section{ACKNOWLEDGMENTS}

This work is supported by the BRGM research directorate in the framework of groundwater modeling and code couplings project.

\section{REFERENCES}

1. K. Aziz and A. Settari, Petroleum Reservoir Simulation, Applied Science, New York, 1979.

2. J. Bear, Dynamics of fluids in porous media, Elsevier, New York, 1972.

3. A. Bellin and Y. Rubin, HYDRO_GEN: A new random field generator for correlated properties. Stochastic Hydrology and Hydraulics, 10(4), 1996.

4. J.E. Garcia and K. Pruess, Flow instabilities during injection of $\mathrm{CO}_{2}$ into saline aquifers, in Proceedings of TOUGH Symposium 2003, Lawrence Berkeley National Laboratory, Berkeley, California, May 12-14, 2003.

5. I.K. Karpov, K.V. Chudnenko and D.A. Kulik, Modeling chemical mass transfer in geochemical processes: thermodynamic relations, conditions of equilibria, and numerical algorithms, Am. J. Sci, 297:767-806, 1997.

6. P.C. Lichtner, Continuum formulation of multicomponent-multiphase reactive transport, in Reactive transport in porous media, P.C. Lichtner, C.I. Steefel and E. H. Oelkers, eds., Reviews in Mineralogy, Mineral. Soc. Am., 34:1-82, 1996.

7. A. Mazzia and M. Putti, Three dimensional Godunov mixed methods on tetrahedra for the advection-dispersion equation, in Proceedings of the XIV ${ }^{\text {th }}$ International Conference on Computational Methods in Water Resources, Developments in Water Science 47(1):381-388, Elsevier, 2002. 
8. J.C. Meza, OPT++: An object-oriented class library for nonlinear optimization, Sandia laboratories report SAND94-8225, 1994.

9. F.M. Morel and J.G. Hering, Principles and applications of aquatic chemistry. John Wiley and Sons, 1993.

10. D.L. Parkhurst and C.A. J. Appelo, User's guide to PHREEQC (version 2) - A computer program for speciation, batch-reaction, one-dimensional transport, and inverse geochemical calculations, Open-File Report 99-4259, USGS, Denver, Colorado, 1999.

11. M.A. Sbai, RTAFF - A new computer model for reactive transport and flow in saturated media, Reference manual and User's guide for version 1.0, BRGM (internal report).

12. M.A. Sbai, M. Azaroual and A. Menjoz, Un nouveau module géochimique de calcul d'équilibre et cinétiques mixtes, BRGM report (to appear), 2004.

13. M.A. Sbai, Modeling three-dimensional groundwater flow and transport by hexahedral finite elements, PhD Thesis, Polytechnical school, Faculty of Applied Sciences, Vrije Universiteit Brussel, VUB-Hydrology 36:211pp, September 1999.

14. C.I. Steefel and K.T. MacQuarrie, Approaches to modeling reactive transport in porous media, in Reactive transport in porous media, P.C. Lichtner, C.I. Steefel and E. H. Oelkers, eds., Reviews in Mineralogy, Mineral. Soc. Am., 34:83-129, 1996.

15. C.I. Steefel and A.C. Lasaga, A coupled model for transport of multiple chemical species and kinetic precipitation/dissolution reactions with application to reactive flow in single phase hydrothermal systems, Am. J. Sci., 294:529-592, 1994.

16. E.F. Toro (ed.), Godunov methods - theory and applications, Academic Publishers, New York, 2001.

17. G.T. Yeh and V.S. Tripathi, A model for simulating transport of reactive multispecies components: model development and demonstration, Water Resour. Res., 27(12):3075-3094, 1991. 